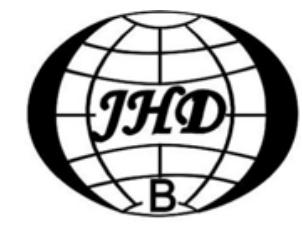

sdlj.chinajournal.net.cn

\title{
INFLUENCE OF CAPILLARITY ON NANO-LITER FLOWRATE MEASUREMET WITH DISPLACEMET METHOD*
}

\author{
GUO Qiang \\ State Key Laboratory of Nonlinear Mechanics, Institute of Mechanics, Chinese Academy of Sciences, \\ Beijing 100080, China \\ Shanghai Institute of Applied Mathematics and Mechanics, Shanghai University, Shanghai 200072, China, \\ E-mail: guoqiangpost@tom.com \\ CHENG Rui, SILBER-LI Zhan-hua \\ State Key Laboratory of Nonlinear Mechanics, Institute of Mechanics, Chinese Academy of Sciences, \\ Beijing 100080, China
}

(Received September 4, 2006; Revised October 13, 2006)

\begin{abstract}
Nano-liter flowrate measurements in micro-tubes with displacement method were performed and the effect of capillarity force on the accuracy was investigated through lab experiments and theoretical analysis in this article. The experiments were conducted under the pressure drops ranging from $1 \mathrm{kPa}$ to $10 \mathrm{kPa}$ in a circular pipe with a diameter of $50 \mu \mathrm{m}$, to give the pressure-flowrate $(P-Q)$ relation and verify the applicability of the classical Hagen-Poiseuille (HP) formula. The experimental results showed that there existed a discrepancy between the experimental data and the theoretical values predicted by the HP formula if the capillary effect was not considered, which exceeded obviously the limit of the system error. And hence a modified formula for the relation, taking the capillary effect into account, was presented through theoretical deduction, and after the HP formula had been modified the error was proved to be less than $3 \%$, which was permitted in comparison with the system error. It was also concluded that only by eliminating the effect of the capillary force in experiments could the original HP formula be employed to predict the pressure-flowrate relation in the Hagen-Poiseuille flow in the micro-tube.
\end{abstract}

KEY WORDS: nano-liter, flowrate measurement, Hagen-Poiseuille (HP) formula, displacement method, capillarity force

\section{INTRODUCTION}

Micro-flow systems have been universally used in various research fields, such as microchemistry analysis, micro-radiator of integrate circuit chips, micro-inject of medicament, ink-spray printers, micro satellite accelerator, liquid-liquid extraction, etc. ${ }^{[1]}$. Research about micro-flow system has been an important subject since the emergence of the Micro Eletro-Mechanical System (MEMS) ${ }^{[2]}$.

Whether the Navier-Stokes equations in hydrodynamics are applicable to micro-flow analysis is always a significant problem, which is still an open problem ${ }^{[3-5]}$. Experimentally determining the pressure-flowrate relation $(P-Q$ relation) for liquid flowing in microtubes, and comparing the results with the prediction of the Hagen-Poiseuille (HP) formula are rather vital for microflow study (the results may also be compared with other experimental data, such as PIV and PTV data $^{[6]}$ ). In the 1990 s, Pfahler et al. ${ }^{[4]}$ and Peng et al. ${ }^{[5]}$ discovered that there were obviously differences between the experimental results and classical results of the HP formula for the $P-Q$ relation. After that, many researchers paid great attentions to this kind of experiments. Li et al. ${ }^{[7]}$ used different liquids to do experiments in quartz micro-tubes with a diameter of $20 \mu \mathrm{m}$. Wang et al. ${ }^{[8]}$ did

\footnotetext{
* Project supported by the National Natural Science Foundation of China (Grant No. 10272107) and Major Innovation Project of Chinese Academy of Sciences(Grant No. KJCX2-SW-L2).

Biography: GUO Qiang (1975- ), Male, Master
} 
researches about the effect of the width of microtube on species diffusion. Maynes et al. ${ }^{[9]}$ carried out experiments in micro-tubes made of different materials, the diameters of their micro-tubes were $15 \mu \mathrm{m}-150 \mu \mathrm{m}$, and the Reynolds number ranged from 8 to 2300. All above experiments verified that the HP formula was still basically valid for micro-tubes. Sharp et al. ${ }^{[10]}$ completed a $P-Q$ experiment with larger diameters $(50 \mu \mathrm{m}-250 \mu \mathrm{m})$ and larger Reynolds number $(R e=1800-2000)$, and reached the same conclusion. Cui et al. ${ }^{[11]}$ used isopropanol and carbon tetrachloride to do $P-Q$ experiments. They found that after a revised HP equation combined with an exponential function of viscosity vs pressure and wall slip were introduced to counteract the difference between experimental and theoretical values, the experiment results were in accordance with the HP prediction. Liu et al. ${ }^{[12]}$ completed an experimental study of microscale flow for micro molecule liquid and polymer solution. They found that water flowing in channels with the diameter equal to or larger than $2 \mu \mathrm{m}$ had few size effects and the wettability did not have influences on the water flow in channels of $25 \mu \mathrm{m}$ or $50 \mu \mathrm{m}$. In most experiments, the inner surface roughness of tubes, evaporation of liquids, heat dissipation and so on were noticed to have great influences on the experimental results. And it is considered that when there are differences between observed flowrate $Q_{\text {exp. and theoretical flowrate } Q_{\text {theo., }} \text { researchers }}$ should not simply think that the NS equations are not valid any more, but oppositely, should analyze the experiment processes carefully, take measures to eliminate the system errors and improve the experiment accuracy. For example, in the experiments of $\mathrm{Qu}{ }^{[13]}$, the friction coefficient $C^{*}$ was larger than theoretically predicted one, which was mainly due to surface roughness and geometry.

The flowrate measurement methods used could be roughly divided into two classes..$^{[7,9-18]}$, i.e., the weighing measurement method ${ }^{[9,10,13,16,18]}$ and the displacement measurement method ${ }^{[7,11,12,14]}$. The uncertainty of weighing measurement relies on the precision of electronic balance (normally $1 \mathrm{mg}$ ), and it fits for the measurement of larger flowrate (normally larger than $1 \mathrm{ml} / \mathrm{s}$ ). The displacement method is a kind of volume method, and according to different displacement precision, fits for the measurement of smaller flowrate (normally smaller than $1 \mu \mathrm{l} / \mathrm{s}$ ). As for $50 \mu \mathrm{m}$ tube with the driving pressure less than $10 \mathrm{kPa}$, the flowrate is at the level of several $\mathrm{nl} / \mathrm{s}$, and hence the displacement method should be adopted. Cheng et al. ${ }^{[14]}$ even measured the flowrate of nano-tube $\left(D_{h}=42 \mathrm{~nm}\right)$ with the displacement method at pressures lower than 120 $\mathrm{kPa}$, and they noticed the influences of capillary of free surface, but did not give detailed experimental data.

In this work, we used an experiment platform which can offer low pressure down to $1 \mathrm{kPa}-10$ $\mathrm{kPa}$ but with a higher precision. We conducted experiments to verify the $P-Q$ relation of $50 \mu \mathrm{m}$ tube with the pressures $\leq 10 \mathrm{kPa}$ and the Reynolds number $\leq 0.4$. The difference between experimental results and theoretical prediction were also observed, that is, $Q_{\text {exp. }} \neq Q_{\text {theo. }}$. After scrupulous analysis, we found the capillarity force was the main factor, and then gave a modified formula to counteract accordingly the effect.

\section{EXPERIMENTAL SETUP AND METHODS}

\subsection{Theoretical results for laminar flow in tubes}

In the case of steady laminar flow in a straight tube, by solving the Navier-Stokes equations the following HP formula can be obtained:

$Q_{\text {theo. }}=\frac{\pi d^{4}}{128 \mu_{0} L} P$

where $d$ and $L$ are the diameter and the length of the micro-tube, respectively, $P$ is the pressure drop between the two ends of the tube, and $\mu_{0}$ the viscosity. The Reynolds number $R e$ and the normalized friction coefficient $C^{*}$ are defined as

$$
\begin{aligned}
& C^{*}=\frac{(\operatorname{Ref})_{\text {exp. }}}{(\operatorname{Ref})_{\text {theo. }}}=\frac{Q_{\text {theo. }}}{Q_{\text {exp. }}} \\
& \operatorname{Re}=\frac{\rho u d}{\mu_{\mathrm{o}}}
\end{aligned}
$$

where $u$ represents the average velocity in the tube, $\rho$ the density of the liquid, $Q_{\text {exp. }}$ the experiment flowrate, and $f$ the friction coefficient.

\subsection{Ultra-low-pressure experimental platform}

The experiments were conducted on an ultra-low- pressure experiment platform (see Fig.1). This platform consists of pressure source, pressure adjustment and control system, pressure and temperature measurement parts, micro-tube experiment section and flowrate measure apparatus. A high pressure nitrogen source can provide pressure up to $15 \mathrm{MPa}$. The pure nitrogen with low 
pressure was obtained through pressure adjustment valves and filters. The pressure adjustment included two steps. The pressure within $100 \mathrm{kPa}$ was obtained through the crude regulation of pressure Sensor I and the pressure lower than $10 \mathrm{kPa}$ was obtained through the fine regulation of pressure Sensor II. Pressure Sensor II and temperature sensor were connected to the tee joint at the inlet end of the micro-tube. When the nitrogen source was switched on, the liquid in the store pipe was put ahead into the micro-tube experiment section. The outlet end of the micro-tube was put into the displacement capillary for the flowrate measurement. The micro-tube was purchased from the Ruifeng Chromatogram Apparatus Co. Its nominal inner diameter was $50 \mu \mathrm{m}$, and the average roughness was less than $3 \%$ as measured by a Scanning Electron Microscope. So the tube could be regarded as the hydraulically smooth tube. The di-water was produced by a super pure water machine of F3SN53133F (Millipore Co). The electrical resistance of the di-water was larger than $0.182 \mathrm{M} \Omega \cdot \mathrm{m}$ at $25^{\circ} \mathrm{C}$.

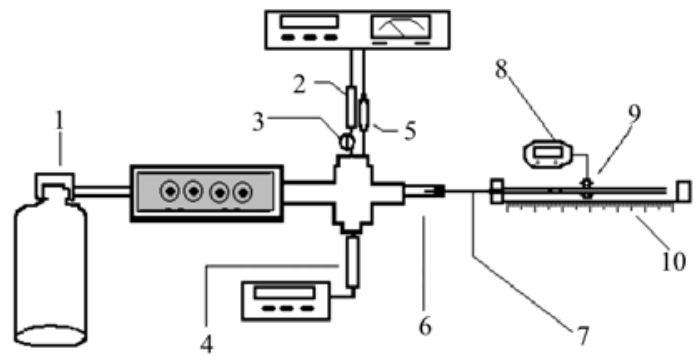

1-High-pressure $\mathrm{N}_{2}$ gas source, 2-Pressure Sensor II, 3-Valve, 4-Pressure Sensor I, 5-Temperature sensor, 6-Liquid storage pipe, 7-Microtube, 8-Interval and displacement display, 9-Photogate sensor, 10-Glass capillary

Fig.1 Sketch of microflow experiment platform

\subsection{Flowrate measurement with displacement method}

Flowrate measurement was performed with the displacement method (see Fig.2). By observing the displacements and time interval $t$ of the free surface of the liquids column in the capillary, the flowrate is calculated as follows:

$Q_{\exp .}=\frac{\pi D^{2}}{4 t} s$

To avoid the evaporation influence of the free surface of liquid, we adopted the method used by
Cui et al. ${ }^{[11]}$ to inject an air bubble into the liquid column in the capillary tube, and the displacement of the bubble interface was recorded by the photogate sensors, rather than the liquid free surface.

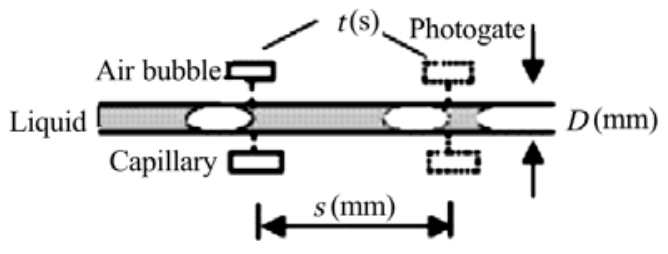

Fig.2 Sketch of flowrate measure with displacement method

\subsection{Experiment uncertainty analysis}

According to the Eqs. (1), (2) and (4), the relative uncertainty of theoretical flowrate $Q_{\text {theo, }}$, experimental flowrate $Q_{\text {exp. }}$ and normalized friction coefficient $C^{*}$ are defined as $\frac{W_{Q_{\text {theo. }}}}{Q_{\text {theo. }}}, \frac{W_{Q_{\text {exp. }}}}{Q_{\text {exp. }}}$ and $\frac{W_{\mathrm{C}^{*}}}{C^{*}}$ respectively.

$$
\begin{aligned}
& \frac{W_{Q_{\text {theo. }}}}{Q_{\text {theo. }}}=\sqrt{\frac{16 W_{d}^{2}}{d^{2}}+\frac{W_{p}^{2}}{P^{2}}+\frac{W_{\mu}^{2}}{\mu_{0}^{2}}+\frac{W_{L}^{2}}{L^{2}}} \\
& \frac{W_{Q_{\text {exp. }}}}{Q_{\text {exp. }}}=\sqrt{\frac{4 W_{D}^{2}}{D^{2}}+\frac{W_{s}^{2}}{s^{2}}+\frac{W_{t}^{2}}{t^{2}}} \\
& \frac{W_{C^{*}}}{C^{*}}=\left(\frac{16 W_{d}^{2}}{d^{2}}+\frac{W_{p}{ }^{2}}{P^{2}}+\frac{W_{\mu}^{2}}{\mu_{0}^{2}}+\frac{W_{L}^{2}}{L^{2}}+\right. \\
& \left.\frac{4 W_{D}^{2}}{D^{2}}+\frac{W_{s}^{2}}{s^{2}}+\frac{W_{t}^{2}}{t^{2}}\right)^{1 / 2}
\end{aligned}
$$

where $W_{i}(i=d, D, p, \mu, L, s, t)$ stand for the experimental uncertainty of the related physical quantities (see Table 1).

By considering the measurement times and relevant standard deviation modification coefficient $\zeta(3 \leq N \leq 5, \quad \zeta=1.32-1.14)$, the total experimental uncertainty of $Q_{\text {theo. }}, Q_{\text {exp. }}$ and $C^{*}$ are given as $\pm 1.76 \%, \pm 1.12 \%$ and $\pm 2.09 \%$ respectively.

\subsection{Process of experiment}

The length of the micro-tube in the experiment is $L=117.56 \mathrm{~mm}$, and the inner diameter $d=57.6 \mu \mathrm{m}$. And the inner diameter of the capillary is 
Table 1 Experimental uncertainty of physical quantities

\begin{tabular}{|c|c|c|c|c|}
\hline Physical quantity & Measurement equipment & Uncertainty & Measurement tube 1 & Value tube 2 \\
\hline$d(\mu \mathrm{m})$ & SEM & \pm 0.1 & 52.6 & 52.7 \\
\hline \multirow[t]{2}{*}{$P(\mathrm{kPa})$} & NS-F6030326 & $\pm 0.3 \%$ & $1.07-9.08$ & $1.01-9.04$ \\
\hline & Ultra-low-pressure sensor & & & \\
\hline$L(\mathrm{~mm})$ & Vernier calipers & \pm 0.02 & 117.56 & 127.56 \\
\hline$T\left({ }^{\circ} \mathrm{C}\right)$ & Temperature sensor & $0.5^{\circ} \mathrm{C}$ & $24.1-24.5$ & 24.1-24.6 \\
\hline$D(\mathrm{~mm})$ & Tool microscope & \pm 0.01 & 1.018 & 1.003 \\
\hline$t(\mathrm{~s})$ & Stopwatch & \pm 0.01 & $96.65-741.06$ & $98.53-770.16$ \\
\hline$s(\mathrm{~mm})$ & $\begin{array}{l}\text { Electronic digital } \\
\text { display calipers }\end{array}$ & \pm 0.01 & 2.00 & 2.00 \\
\hline
\end{tabular}

$D=1.018 \mathrm{~mm}$. When the driving pressure drop is lessthan $10 \mathrm{kPa}$ and the temperature is about $24^{\circ} \mathrm{C}$, the Reynolds number $R e \leq 0.4$. The pressure in experiments was adjusted from low to high, and at each pressure value, three flowrate values were recorded. If the relative deviations among one set of observed flowrate values are larger than 5\%, another new set of observations would be conducted again, till a set of stable results had been obtained.

\section{EXPERIMENTAL RESULTS}

\subsection{Eexperiments for pressure-flowrate relation in a} 50 um tube

In Fig.3, experimental results (triangle points) and theoretically predicted results (straight line) are shown. Two dashed lines besides the straight line give the uncertainty range of the theoretical predictions.

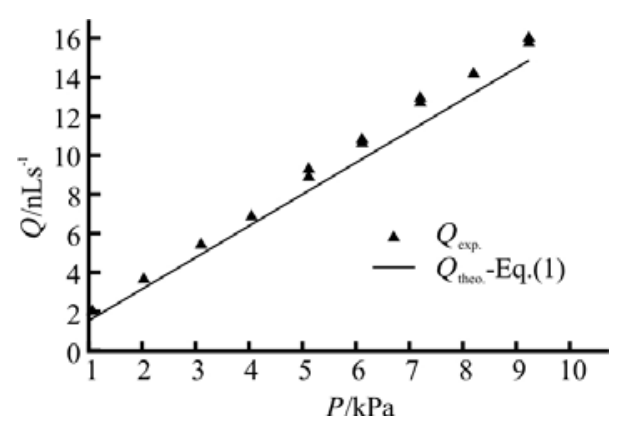

Fig.3 $P-Q$ relation obtained in a $50 \mu \mathrm{m}$ tube under ultra-low-pressure and comparison with theoretical prediction

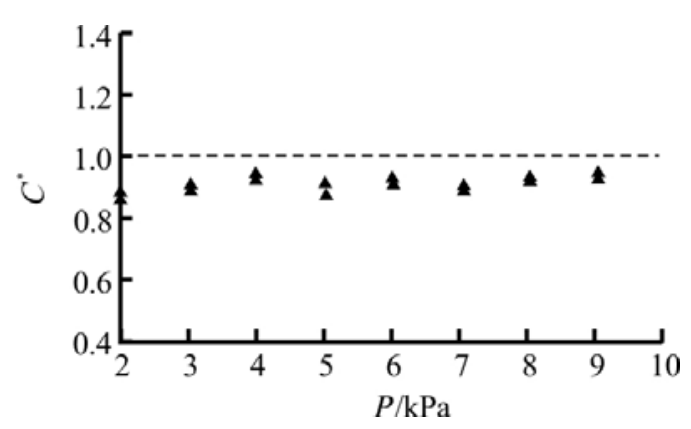

Fig.4 $P-C^{*}$ relation obtained in a $50 \mu \mathrm{m}$ tube under ultra-low-pressure and comparison with theoretical prediction

Figure 4 gives the $C^{*}$-pressure relation. As shown in Fig.3, the experimental data $Q_{\text {exp. }}$ are $5 \%-10 \%$ larger than $Q_{\text {theo. }}$. We used another tube to repeat the experiment $(d=52.7 \mu \mathrm{m}, L=127.56 \mathrm{~mm})$, and the results were similar. However, previous studies have shown that the experimental $P-Q$ relation in a $50 \mu \mathrm{m}$ tube is in accordance with theoretical prediction ${ }^{[9,10]}$. Why are there differences in ultra-low-pressure micro-flow experiment $(0 \mathrm{kPa}-10 \mathrm{kPa})$ ? We will try to answer this question below.

3.2 Pressure drop analysis in the micro-tube and the displacement tube

As shown in Fig.5, we suppose that the pressure value is $P_{1}$ at the inlet of micro-tube, $P_{2}$ at the outlet, $P_{3}$ at the free surface of liquid in the capillary, and $P_{0}$ in the atmospheric pressure. 


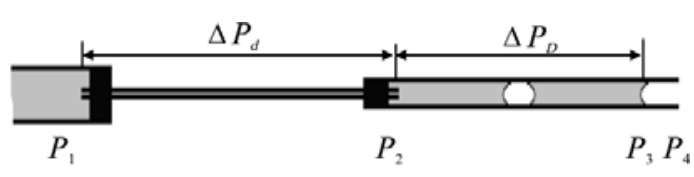

Fig.5 Sketch of pressure distribution in micro-tube and displacement tube

Using the theoretical flowrate formula (1) and flowrate conservation principle, we have $D^{4} L \triangle P_{D}=d^{4} l \triangle P_{d}$. The inner diameter and length of the micro-tube are $d=52.6 \mu \mathrm{m}, L=127.56 \mathrm{~mm}$, and those for the capillary tube, $D=1.018 \mathrm{~mm}$, $l=150.03 \mathrm{~mm}$, so we have

$$
\frac{\Delta P_{d}}{\Delta P_{D}}=\frac{D^{4} L}{d^{4} l} \square 10^{4}
$$

Therefore, the pressure drop between the two ends of the capillary can be ignored, that is, $\triangle P_{D} \approx 0$. If we ignore also the capillarity force at the free surface of liquid, and then considering $P_{0}=P_{3}$, we have $\triangle P_{d}=P_{1}-P_{0}$, which is the reading of the pressure Sensor II.

As there is capillarity force at the liquid free surface in the capillary, we need to calculate its actual value. At the temperature of $24^{\circ} \mathrm{C}$ in the experiment, the surface tension coefficient of pure water is $71.95 \mathrm{~mJ} / \mathrm{m}^{2}$. After having measured the contact angle in the capillary we adopted its average value of $20^{\circ}$. The inner diameter of the capillary is $1.018 \mathrm{~mm}$ and the capillarity force $P_{c}$ can be calculated according to the Laplace formula ${ }^{[19]}$, given as

$P_{c}=\frac{2 \gamma \cos \theta}{R} \approx 0.27 \mathrm{kPa}$

Taking into account the capillarity force at the free surface, we have $P_{3}=P_{0}-P_{\mathrm{c}}$, and

$$
\begin{gathered}
\Delta P_{d}^{\prime}=P_{1}-P_{2} \approx P_{1}-P_{3}=P_{1}-\left(P_{0}-P_{c}\right)= \\
\Delta P_{d}+P_{c}
\end{gathered}
$$

So the theoretical formula for the flowrate can be modified to be

$$
\begin{gathered}
Q_{\text {theo. }}^{\prime}=\frac{\pi d^{4}}{128 \mu_{0} L}\left(P+P_{c}\right)=Q_{\text {theo. }}+ \\
\frac{\pi d^{4}}{128 \mu_{0} L} P_{c}
\end{gathered}
$$

It can be seen from Eq. (10) that, if the micro-tube pressure drop $\triangle P_{d}$ is much greater than the capillarity force $P_{c}$, then $\triangle P_{d}^{\prime} \approx \triangle P_{d}$, that is, the capillarity force can be ignored. This situation appeared in the experiments of $\mathrm{Li}^{[7]}$ and $\mathrm{Cui}^{[11]}$. Although the displacement method was adopted, the driving pressure drop was very large, and the capillarity force needs not to be considered. For the case studied herein, the driving pressure drop is ultra-low, $\triangle P_{d}=1 \mathrm{kPa}-9 \mathrm{kPa}$, and the capillarity force $P_{c} \approx 0.3 \mathrm{kPa}$, so the influence of $P_{c}$ may reach a fraction of $3 \%-30 \%$, which can not be ignored.

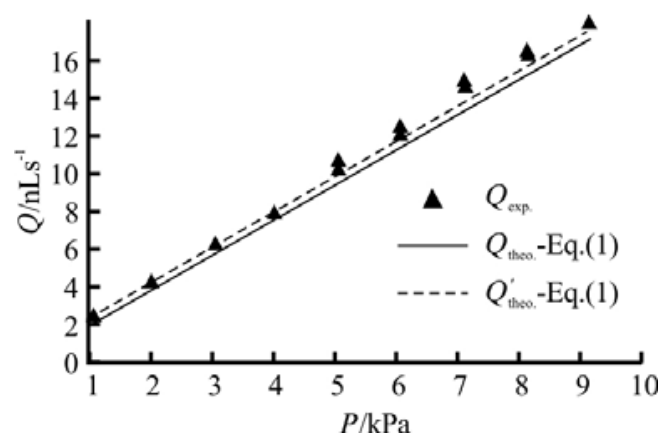

Fig.6 $P-Q$ relation obtained in a $50 \mu \mathrm{m}$ tube and comparison with revised theoretical prediction

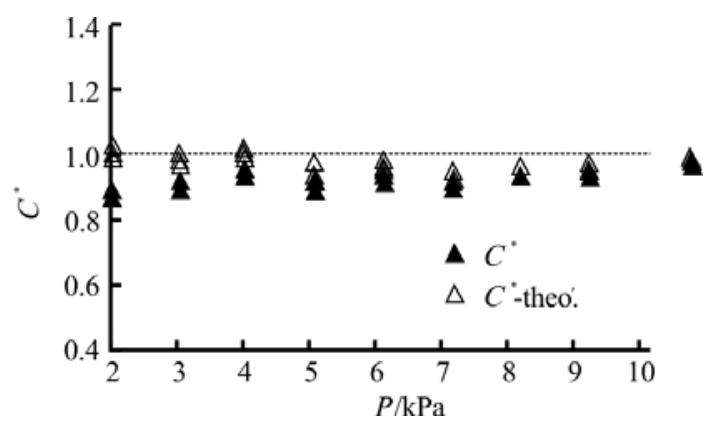

Fig.7 $P$ - $C^{*}$ relation obtained in a $50 \mu \mathrm{m}$ tube and comparison with revised theoretical prediction

\subsection{Conparison with experimental results}

In Fig.6, the curve (dashed line) obtained from the revised flowrate formula, Eq. (11), is given. 
Obviously, it is in better accordance with experimental results (triangle point), and the error is less than $3 \%$. At the same time, $C^{*}$ tends to be 1 after the modification (see Fig.7).

\subsection{Experimental verification of influence of} capillarity force

To confirm the influences brought about by the capillarity force at the free surface of liquid, we filled the capillary downstream the air bubble with water (see Fig.8), and repeated the same experiment.

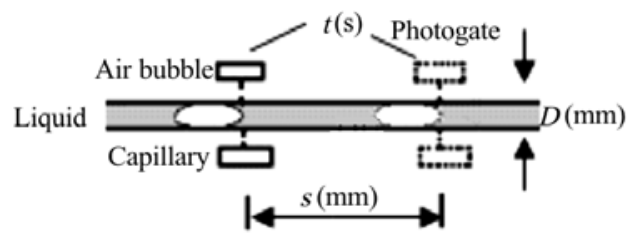

Fig.8 Filling the downstream capillary section of the air bubble with water

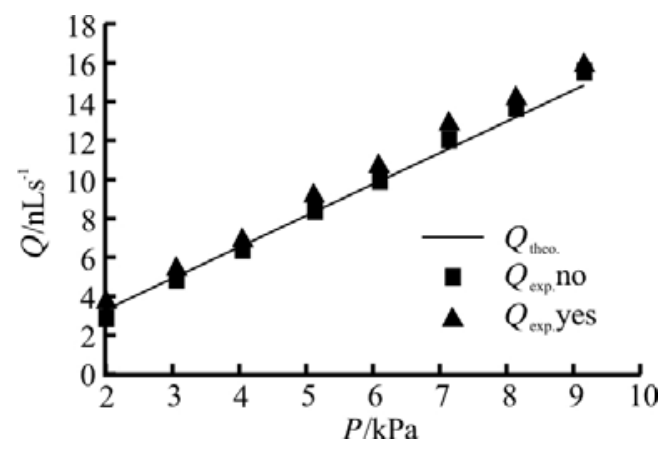

Fig.9 $P-Q$ relation obtained in a $50 \mu \mathrm{m}$ tube with and without the capillarity force

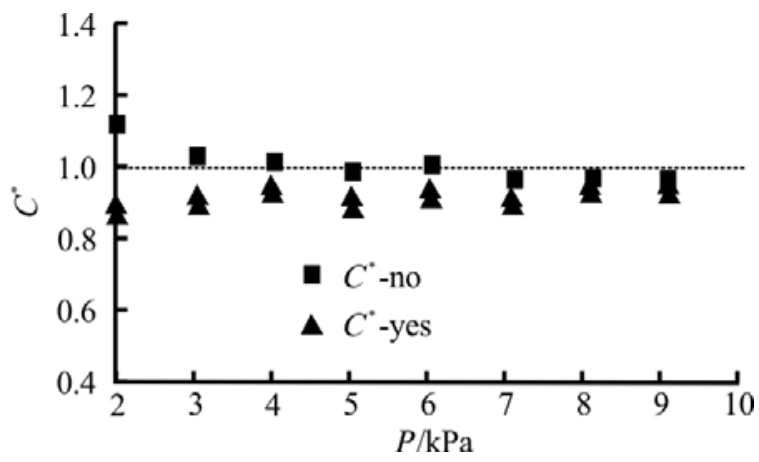

Fig.10 $P$ - $C^{*}$ relation obtained in a $50 \mu \mathrm{m}$ tube with and without the capillarity force

The experimental results are illustrated in Figs.9 and 10. It is seen from Fig.9 that after the capillarity force is eliminated, experimental values of flowrate are closer to the theoretical values than those in the former experiment. From Fig.10, it can be seen that $C^{*}$ approaches nearly to 1 .

\section{CONCLUSIONS}

With the ultra-low-pressure platform built in the LNM, Institute of Mechanics, Chinese Academy of Sciences, the flow characteristics of di-water in a $50 \mu \mathrm{m}$ tube under $0 \mathrm{kPa}-10 \mathrm{kPa}$ pressure have been investigated. It was found that the experimental values of both flowrate and normalized friction coefficient $C^{*}$ have 5\%-10\% deviation from theoretical prediction.

Through analyzing the influence of the capillarity force at the free surface of the liquid in the capillary, it was concluded that the capillarity force can cause errors up to $3 \%-30 \%$. The effect of the capillarity force has been introduced to modify the HP flowrate formula, and the modified results are in good accordance with the experiment results with the average error less than $3 \%$. In another verification experiment, it has been shown that eliminating the free surface of liquid column in the capillary can avoid the effects of the capillarity force.

Therefore the results in this article indicate that the capillarity force has a strong influence in nano-liter flowrate measurement under ultralow-pressure, and the related calculation formula need to be modified, or else, if the classical HP formula is required to be used, the capillarity force should be eliminated in experiments.

\section{ACKNOWLEDGEMENT}

The authors would like to give their heartfelt thanks to Prof. Dai Shi-qiang of Shanghai University for his help in preparing the manuscript.

\section{REFERENCES}

[1] BAO Fu-bing, LIN Jian-zhng and LIU Yan-hua. Research on the flow property in three dimensional cavity of micro-channel [J]. Journal of Hydrodynamics, Ser. B, 2006, 18(1): 20-25.

[2] HO Chih-Ming, TAI Yu-Chong. Micro-electromechanical-systems (MEMS) and fluid flows [J]. Annu. Rev. Fluid Mech., 1998, 30: 579-612.

[3] DING Ying-tao, HE Feng and YAO Zhao-hui et al. Sub-choking phenomenon of low-speed gas flow in a long-constant-area microchannel transport in small channels [J]. Acta Physics Sinica, 2004, 53(8): 2429-2433(in Chinese).

[4] PFAHLER J., HARLEY J. and BAU H. H. et al. Liquid and gas transport in small channels [J]. DSC, 1990, 19: 149-157.

[5] PENG X. F., PETERSON G. P. and WANG B. X. Frictional flow characteristic of water flowing through rectangular micro-channels [J]. J. Exp. Heat Transfer, 1995, 7(4): 249-264. 
[6] CHENG Wen, MURAL Yuichi and SASAKI Toshio et al. Liquid phase flow estimation in gas-liquid two-phase flow using inverse analysis and particle tracking velocimetry[J]. Journal of Hydrodynamics, Ser. B, 2004, 16(6): 730-736.

[7] LI Zhan-hua, ZHOU Xing-bei and ZHU Shan-nong. Flow characteristics of non-polarity small molecule organic liquid in micro-tubes [J]. Acta Mechanica Sinica, 2002, 34(3):432-438 (in Chinese).

[8] WANG Rui-jin, LIN Jian-zhong. Numerical simulation of transverse diffusion in a micro- channel $[\mathrm{J}]$. Journal of Hydrodynamics, Ser. B, 2004, 16(6): 651-657.

[9] MAYNES J. D., WEBB B.W. Characterization of frictional pressure drop for liquid flows through microchannels [J]. Int. J. Heat Mass Transfer, 2002, 45(17): 3477-3489.

[10] SHARP K.V., ADRIAN R. J. Transition from laminar to turbulent flow in liquid filled micro-tubes [J]. Exp. Fluids, 2004, 36(5): 741-747.

[11] CUI Hai-Hang,SILBER-LI Zhan-hua. Flow characteristics of liquids in microtubes driven by a high pressure [J]. Physics Fluids, 2004, 16(5): 1803-1810.

[12] LIU Zhong-chun, YUE Xiang-an and HOU Ji-rui et al. Experimental study of microscale flow for micro molecule liquid and polymer solution $[\mathrm{J}]$. Journal of Hydrodynamics, Ser. B, 2005, 17(3): 352-357.
[13] QU Wei-lin, MALA G. M. and LI Dong-qing. Pressure-driven water flows in trapezoidal silicon micro-channels [J]. Int. J. Heat Mass Transfer, 2000, 43(3): 353-364.

[14] CHENG J. T. , GIODANO N. Fluid flow through nanometer-scale channels [J]. Phys. Rev. E, 2002, 65(3): $1-5$.

[15] MAYNES D., WEBB A. R. Velocity profile characteristic in sub-diameter tubes using molecular tagging velocimeter [J]. Exp. Fluids, 2002, 32(1): 3-15.

[16] WU H. Y., CHENG P. Friction factors in smooth trapezoidal silicon micro-channels with different aspect ratios [J]. Int. J. Heat Mass Transfer, 2003, 46(14): 2519-2525.

[17] LELEA D., NISHIIO S. and TAKANO K. The experimental research on micro-tube heat transfer and fluid flow of distilled water[J]. Int. J. Heat Mass Transfer, 2004, 47(12): 2817-2830.

[18] CHOI Chang-Hwan, JOHAN K. and KENNETH A. W. et al. Apparent slip flows in hydrophilic and hydrophobic micro-channels [J]. Phys. Fluids, 2003, 15(10): 2897-2902.

[19] GU Xi-ren, ZHU Bu-yao et al. Surface chemistry[M]. Beijing: Science Press, 2001, 13-15 (in Chinese). 